\title{
OSCILLATION CRITERIA FOR SECOND ORDER NONLINEAR DIFFERENTIAL EQUATIONS
}

\section{HIROSHI ONOSE}

ABSTRACT. Oscillation criteria are given for the second order nonlinear equation $y^{\prime \prime}(t)+a(t) f(y(t))=0$, where the coefficient $a(t)$ is not assumed to be nonnegative for all large values of $t$. These results are concerned with the interesting recent ones of Wong's paper.

1. Introduction. Consider the second order nonlinear differential equation

$$
y^{\prime \prime}(t)+a(t) f(y(t))=0
$$

and its special case

$$
y^{\prime \prime}(t)+a(t)|y|^{\gamma} \operatorname{sgn} y=0, \quad \gamma>0
$$

where $a(t) \in C[0, \infty)$. We consider only those solutions of (1) which exist on $\left[t_{0}, \infty\right)$. A solution of (1) is said to be oscillatory if it has arbitrarily large zeros. Equation (1) is called oscillatory if all such solutions are oscillatory.

For simplicity, we mention the conditions used in the following sections:

$$
\begin{gathered}
f^{\prime}(x)>k>0 \text { for all } x \geq 0 \\
\int_{\epsilon}^{\infty} \frac{d u}{f(u)}<\infty \quad \text { for some } \epsilon>0
\end{gathered}
$$

and

$$
\begin{gathered}
\int_{-\epsilon}^{-\infty} \frac{d u}{f(u)}<\infty \text { for some } \epsilon>0 \\
\int_{0}^{\epsilon} \frac{d u}{f(u)}<\infty \quad \text { for some } \epsilon>0
\end{gathered}
$$

and

Received by the editors February 25, 1974 and, in revised form, May 21, 1974. AMS (MOS) subject classifications (1970). Primary 34C10, 34C15.

Key words and phrases. Second order equations, nonlinear, oscillatory. 


$$
\int_{0}^{-\epsilon} \frac{d u}{f(u)}<\infty \text { for some } \epsilon>0
$$

$$
\begin{gathered}
\lim _{T \rightarrow \infty} \int_{0}^{T} a(s) d s=+\infty, \\
\lim _{T \rightarrow \infty} \frac{1}{T} \int_{0}^{T} \int_{0}^{t} a(s) d s d t=+\infty, \\
\lim _{T \rightarrow \infty} \sup \frac{1}{T} \int_{0}^{T} \int_{0}^{t} a(s) d s d t=+\infty \\
\lim _{T \rightarrow \infty} \inf \int_{0}^{T} a(s) d s>-\lambda>-\infty, \lambda>0, \\
\lim _{T \rightarrow \infty} \inf \int_{0}^{T} a(s) d s \geq 0, \\
\lim _{T \rightarrow \infty} \sup \int_{0}^{T} \int_{0}^{t} a(s) d s d t=+\infty \\
\lim _{T \rightarrow \infty} \sup \int_{0}^{T} a(s) d s=+\infty .
\end{gathered}
$$

Recently, J. S. W. Wong [12] proposed a very interesting problem and provided a partial solution to it. Many oscillation criteria for (1) and (2) under conditions (6) or (10) have been obtained; refer to [1]-[5], [7]-[9] and [11]. Now Wong's [12] problem is whether the weaker hypothesis (7) is sufficient for the oscillation of (2) for all $\gamma>0$ and also for (1). Recently, Kamenev [6] proved conditions (5) and (8) suffice for the oscillation of (1). And also Wong [12] proved for $\gamma>1$ that conditions (8) and (9) suffice for the oscillation of (2).

In this paper we prove a theorem of Wong's type for the sublinear case and also study the extension of Wong's result to the more general superlinear case (1).

\section{Oscillation theorems.}

Theorem 1. Suppose that conditions (9), (11) and (12) bold. Then equation (2) is oscillatory for $0<\gamma<1$.

Proof. Assume the contrary; then there exists a solution $y(t)$ which may be assumed to be positive on $\left[t_{0}, \infty\right)$ for some $t_{0} \geq 0$. We distinguish three cases for the behavior of $y^{\prime}(t)$ : 
(i) $y^{\prime}(t)$ oscillatory on $\left[t_{0}, \infty\right)$,

(ii) $y^{\prime}(t)>0$ on $\left[t_{*}, \infty\right)$ for some $t_{*} \geq t_{0}$,

(iii) $y^{\prime}(t)<0$ on $\left[t_{*}, \infty\right)$ for some $t_{*} \geq t_{0}$.

Suppose case (i) holds; then there exists a sequence $\left\{t_{n} ; n=1,2, \cdots\right\}$ such that $y^{\prime}\left(t_{n}\right)=0$ and $t_{n} \rightarrow \infty$. Dividing (2) through by $y^{\gamma}(t)$ and integrating from $t_{k}$ to $t$, we obtain

$$
y^{-\gamma}(t) y^{\prime}(t)+\gamma \int_{t_{k}}^{t}\left(\frac{y^{\prime}(s)}{y^{\beta}(s)}\right)^{2} d s+A(t)=0, \quad A(t) \equiv \int_{t_{k}}^{t} a(s) d s,
$$

where $\beta=(\gamma+1) / 2$ and $y^{\prime}\left(t_{k}\right)=0$ ( $k$ is some integer). Integrating (13) once more from $t_{k}$ to $t$, we obtain

$$
\frac{1}{1-\gamma} y^{1-\gamma}(t)+\gamma \int_{t_{k}}^{t} \int_{t_{k}}^{s}\left(\frac{y^{\prime}(u)}{y^{\beta}(u)}\right)^{2} d u d s+\int_{t_{k}}^{t} A(s) d s=\frac{y^{-\gamma+1}\left(t_{k}\right)}{1-\gamma}
$$

From (14), we have a contradiction to condition (11).

Next suppose that $y^{\prime}(t)>0$ for $t \geq t_{1} \geq t_{0}$; thus $y(t)>y\left(t_{1}\right)$. Dividing (1) through by $y^{\gamma}(t)$ and integrating from $t_{*}\left(\geq t_{1}\right)$ to $t$, we obtain

$$
y^{-\gamma}(t) y^{\prime}(t)+\gamma \int_{t_{*}}^{t}\left(\frac{y^{\prime}(s)}{y^{\beta}(s)}\right)^{2} d s+\int_{t_{*}}^{t} a(s) d s=c_{1},
$$

where $c_{1}=y^{\prime}\left(t_{*}\right) / y^{\gamma}\left(t_{\dot{*}}\right)$. This leads to a contradiction to (12).

Finally, we assume that $y^{\prime}(t)<0$ for $t \geq t_{*} \geq t_{0}$. By condition (9) we can estimate (15) as follows (in this case (15) is also valid)

$$
-\frac{y^{\prime}(t)}{y^{\gamma}(t)} \geq-\left(c_{1}+\lambda\right)+\gamma \int_{t_{*}}^{t} \frac{\left(y^{\prime}(s)\right)^{2}}{y^{\gamma+1}(s)} d s .
$$

If the integral in (16) is finite, namely

$$
\lim _{t \rightarrow \infty} \gamma \int_{t_{*}}^{t} \frac{\left(y^{\prime}(s)\right)^{2}}{y^{\gamma+1}(s)} d s \leq M \quad(M \text { is constant })
$$

then we have

$$
\lim _{t \rightarrow \infty} \inf \frac{\left(y^{\prime}(t)\right)^{2}}{y^{\gamma+1}(t)}=\lim _{t \rightarrow \infty} \inf \left(\frac{-y^{\prime}(t)}{y^{\beta}(t)}\right)^{2}=0
$$

From this and $y^{\prime}(t)<0$, we conclude that 


$$
\lim _{t \rightarrow \infty} \inf \frac{-y^{\prime}(t)}{y^{\beta}(t)}=0
$$

As $y^{\prime}(t)<0$, we have $\lim _{t \rightarrow \infty} y(t)=k \geq 0$. Taking first the case $k=0$, we have $\beta-\gamma=(1-\gamma) / 2>0, y(t)<1$ and

$$
\left(-\frac{y^{\prime}(t)}{y^{\beta}(t)}\right)-\left(-\frac{y^{\prime}(t)}{y^{\gamma}(t)}\right)=\left(\frac{y^{\beta}(t)-y^{\gamma}(t)}{y^{\beta}(t) y^{\gamma}(t)}\right) y^{\prime}(t)>0,
$$

for sufficiently large $t$. From (16) and (19), we have

$$
-\frac{y^{\prime}(t)}{y^{\beta}(t)}>-\frac{y^{\prime}(t)}{y^{\gamma}(t)}>-c_{1}-\lambda, \quad c_{1}=\frac{y^{\prime}\left(t_{*}\right)}{y^{\gamma}\left(t_{*}\right)} .
$$

From (18) and (20), we have

$$
0 \geq-c_{1}-\lambda, \text { i.e. } \lambda \geq-c_{1} .
$$

If $\lim _{t \rightarrow \infty}$ inf $y^{\prime}(t) / y^{\gamma}(t)>-\infty$, then by (15) we have a contradiction to hypothesis (12). So we must have $\lim _{t \rightarrow \infty}$ inf $y^{\prime}(t) / y^{\gamma}(t)=-\infty$. In this case we have a contradiction to (21) because we can take $t_{*}$ such that the absolute

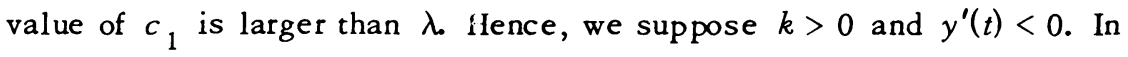
this case we have

$$
k \leq y(t) \leq k+1 \text { for sufficiently large } t .
$$

From (22) and (17) we obtain

$$
\lim _{t \rightarrow \infty} \int_{t_{*}}^{t}\left(y^{\prime}(s)\right)^{2} d s \leq N \quad(N \text { is some constant }) .
$$

From (23) we conclude

$$
\lim _{t \rightarrow \infty} \inf \left(y^{\prime}(t)\right)^{2}=0
$$

From $\lim _{t \rightarrow \infty} y(t)=k>0$ and (16) we have

$$
-y^{\prime}(t) / k^{\gamma} \geq-y^{\prime}(t) / y^{\gamma}(t) \geq-c_{1}-\lambda,
$$

so that this fact and (24) imply

$$
0 \geq-c_{1}-\lambda
$$

From (25) and the argument used previously, we also have a contradiction. Hence, we suppose 


$$
\lim _{t \rightarrow \infty} \gamma \int_{t_{*}}^{t} \frac{\left(y^{\prime}(s)\right)^{2}}{y^{\gamma+1}(s)} d s=\infty
$$

In this case we may choose $T \geq t_{*}$ such that

$$
\gamma \int_{t_{*}}^{T} \frac{\left(y^{\prime}(s)\right)^{2}}{\gamma+1(s)} d s=1+c_{1}+\lambda
$$

For $t \geq T_{1}$, we multiply (16) through by

$$
-y^{\prime}(t) /\left[y(t)\left\{-\left(c_{1}+\lambda\right)+\gamma \int_{t_{*}}^{t} \frac{\left(y^{\prime}(s)\right)^{2}}{y^{\gamma+1}(s)} d s\right\}\right]
$$

and integrate from $T$ to $t$ to obtain

$$
\log \left[-\left(c_{1}+\lambda\right)+\gamma \int_{t_{*}}^{t} \frac{\left(y^{\prime}(s)\right)^{2}}{y^{\gamma+1}(s)} d s\right] \geq \gamma \int_{T}^{t} \frac{\left[-y^{\prime}(s)\right]}{y(s)} d s
$$

which together with (16) yields

$$
-y^{\prime}(t) / y^{\gamma}(t) \geq y^{\gamma}(T) / y^{\gamma}(t) .
$$

It follows from (27) that $y^{\prime}(t) \leq-y^{\gamma}(T)<0$, which contradicts the as sumption that $y(t)>0$.

Theorem 2. Suppose that (10) and (12) hold. Then, equation (1) is oscillatory.

The proof of Theorem 2 is obtained by closely looking at the proof of Theorem 1.

Theorem 3. Suppose that (3), (4), (8) and (9) bold. Then, equation . (1) is oscillatory.

Proof. Suppose that a solution $y(t)$ of (1) is nonoscillatory; then $y(t)$ may be assumed to be positive on $\left[t_{0}, \infty\right)$ for some $t_{0} \geq 1>0$. Dividing (1) through by $f(y(t))$ and integrating it twice, we obtain

$$
\frac{y^{\prime}(t)}{f(y(t))}+\int_{t_{0}}^{t} f^{\prime}(y(s))\left[\frac{y^{\prime}(s)}{f(y(s))}\right]^{2} d s+A(t)=c_{1},
$$

and

$$
\int_{t_{0}}^{t} \frac{y^{\prime}(s)}{f(y(s))} d s+\int_{t_{0}}^{t} \int_{t_{0}}^{t} f^{\prime}(y(s))\left[\frac{y^{\prime}(s)}{f(y(s))}\right]^{2} d s d t+\int_{t_{0}}^{t} A(s) d s=c_{1} t+c_{2},
$$


where $c_{1}, c_{2}$ are constant and $A(t)=\int_{t_{0}}^{t} a(s) d s$. We distinguish three cases of the behavior of $y^{\prime}(t)$, namely, (i), (ii), (iii) of Theorem 1. Suppose case (i) holds; then there exists a sequence $\left\{t_{n} ; n=1,2, \cdots\right\}$ such that $y^{\prime}\left(t_{n}\right)=0$ and $t_{n} \rightarrow \infty$. From this fact, (28) and (9), we have that

$$
\int_{t_{0}}^{t} f^{\prime}(y(s))\left[\frac{y^{\prime}(s)}{f(y(s))}\right]^{2} d s \text { is finite. }
$$

From (30) and (3) we see that

$$
\int_{t_{0}}^{t}\left[\frac{y^{\prime}(s)}{f(y(s))}\right]^{2} d s \leq N^{2} \quad(N \text { is a positive constant }) .
$$

By Schwarz's inequality we have

$$
\begin{aligned}
\left|\int_{y\left(t_{0}\right)}^{y(t)} \frac{d s}{f(s)}\right|^{2} & =\left|\int_{t_{0}}^{t} \frac{y^{\prime}(s)}{f(y(s))} d s\right|^{2} \\
& \leq t \int_{t_{0}}^{t}\left[\frac{y^{\prime}(s)}{f(y(s))}\right]^{2} d s \leq t^{2} N^{2} \quad\left(t \geq t_{0} \geq 1\right) .
\end{aligned}
$$

From (29) and (32) we have a contradiction to condition (8).

Suppose case (ii) holds, namely $y^{\prime}(t)>0$; then from conditions (4) and (29) we again have a contradiction to condition (8). Finally, assume that $y^{\prime}(t)<0$. By (9) and (28) we have

$$
-\frac{y^{\prime}(t)}{f(y(t))} \geq-\left(c_{1}+\lambda\right)+\int_{t_{0}}^{t} f^{\prime}(y(s))\left[\frac{y^{\prime}(s)}{f(y(s))}\right]^{2} d s .
$$

If the right integral in (33) is finite as $t \rightarrow \infty$, then we arrive at a contradiction by the procedure of case (i). Otherwise, by multiplying (33) through by

$$
\frac{f^{\prime}(y(t)) y^{\prime}(t)}{f(y(t))} /\left\{-\left(c_{1}+\lambda\right)+\int_{t_{0}}^{t} f^{\prime}(y(s))\left[\frac{y^{\prime}(s)}{f(y(s))}\right]^{2} d s\right\}
$$

and by employing the same procedure as in the proof of Theorem 1, we have $y^{\prime}(t) \leq-f(y(T)) \leq 0$, which leads to a contradiction to the assumption $y(t)>0$.

Remark. For the equation $y^{\prime \prime}(t)+a(t)\left(y(t)^{3}+y(t)\right)=0$, Theorem 3 is valid, but Wong's [12] theorem cannot be applied. 
For the case without condition (3), we also have, by the argument of Theorem 3,

Theorem 4. Suppose that (4), (8) and (9) bold. Then every solution of (1) is oscillatory, or $\lim _{t \rightarrow \infty}|y(t)|=0$, or $\lim _{i \rightarrow \infty}|y(t)|=0$ with its derivative $y^{\prime}(t)$ being oscillatory.

Acknowledgment. The author wishes to express his thanks to the referee for some useful comments.

\section{REFERENCES}

1. N. P. Bhatia, Some os cillation theorems for second order differential equations, J. Math. Anal. Appl. 15 (1966), 442-446. MR 34 \#3017.

2. L. E. Bobisud, Oscillation of nonlinear second order equations, Proc. Amer. Math. Soc. 23 (1969), 501-505. MR 40 \#48.

3. W. J. Coles, An oscillation criterion for second order linear differential equations, Proc. Amer. Math. Soc. 19 (1968), 755-759.

4. L. Erbe, Oscillation theorems for second order nonlinear differential equa. tions, Proc. Amer. Math. Soc. 24 (1970), 811-814. MR 40 \#5973.

5. P. Hartman, On non-oscillatory linear differential equations of second order, Amer. J. Math. 74 (1952), 389-400. MR 14, 50.

6. I. V. Kamenev, Certain specifically nonlinear oscillation theorems, Mat. Zametki 10 (1971), 129-134 = Math. Notes 10 (1971), 502-505. MR 44 \#4284.

7. W. Leighton, The detection of the oscillation of solutions of a second order linear differential equation, Duke Math. J. 17 (1950), 57-61. MR 11, 248; 11, 871.

8. H. Onose, On oscillation of nonlinear second order equations, J. Math. Anal. Appl. 39 (1972), 122-124.

9. P. Waltman, An oscillation criterion for a nonlinear second order equation, J. Math. Anal. Appl. 10 (1965), 439-441. MR 30 \#3265.

10. A. Wintner, A criterion of os cillatory stability, Quart. Appl. Math. 7 (1949), 115-117. MR 10, 456 .

11. J. S. W. Wong, On two theorems of Waltman, SIAM J. Appl. Math. 14 (1966), 724-728. MR 34 \#6228.

12. - A second order nonlinear oscillation theorem, Proc. Amer. Math. Soc. 40 (1973), 487-491. MR $47 \# 7132$. 